\title{
Review of: "QTLs Detection for Low-Temperature Germination in Rapeseed by QTL-seq and Linkage Mapping Approach"
}

\author{
Yawen Zeng ${ }^{1}$ \\ 1 Yunnan Academy of Agriculture Sciences
}

Potential competing interests: The author(s) declared that no potential competing interests exist.

\author{
Yawen Zeng ${ }^{1}$ \\ 1 Post Scientist; Biotechnology and Germplasm Resources Institute of Yunnan Academy of Agricultural \\ Sciences, Kunming 650205, China
}

Low temperature is a major factor limiting crop productivity and geographical distribution. Both oil (Rapeseed) and food (rice, barley) for major crop in the world are susceptible to low-temperature stress, which delays seed germination and increases seedling mortality, adversely afecting crop growth and production. This article reveals that the genetic mechanisms of seed germination under low temperatures in rapeseed based on a total of $574 \mathrm{~F}_{2: 3}$ families of derived from 3429×Huyou21 using the QTL-seq approach and linkage analysis of the populations: a total of 319,059,584 reads were generated for low tolerant bulk and 23,983,156 reads for tolerant parent (Huyou21), 335,343,844 reads for low sensitive bulk and 337,990,498 reads for susceptible parent (3429); 5,600,575 genome-wide SNPs and 1,215,792 InDels were obtained for low tolerant bulk and 5,604,534 SNPs and 1,217,972 InDels for low sensitive bulk by comparing with the reference genome; One qLTGA9-1 was mapped to a $341.86 \mathrm{~kb}$ interval with 69 genes between the SSR markers Nys9A212 and Nys9A215 on rapeseed chromosome A09; Another qLTGC1-1 was mapped to a 1.31 Mb interval with 133 genes between the SSR markers Nys1C96 and Nys1C117 on chromosome C01[1]. The Ningyou 7 identifed that seven of the predicted genes encode proteins associated with plant growth or temperature stress response but three genes (one genes in qLTGA9-1 and two genes in qLTGC1-1) no annotation (See table 1). These results provide a basis for genetic breeding and genes cloning for cold tolerance in rapeseed, It also develops new ideas for the gene excavation of cold tolerance and its molecular breeding during crop germination period.

Table 1. The candidate genes with functional efect variants in qLTGA9-1 and qLTGC1-1 Regions [1] 


\begin{tabular}{|c|c|c|c|c|c|}
\hline QTL & Gene ID & Start base-end base (bp) & Functional efect & Chain & Predicted function \\
\hline \multirow{7}{*}{ qLTGA9-1 } & ChrA09g005501 & 44842926-44843909 & Moderate & Forward & Formiminotransferase \\
\hline & ChrA09g005502 & $44845212-44845795$ & Moderate & Reverse & - \\
\hline & ChrA09g005507 & $44864501-44865259$ & Moderate & Forward & Protein phosphatase $2 \mathrm{C}$ \\
\hline & ChrA09g005509 & $44869664-44570813$ & High & Forward & Aminotransferase class I and II \\
\hline & ChrA09g005523 & 44950207-44952401 & Moderate & Forward & DRG Family regulatory protein \\
\hline & ChrA09g005524 & $44954947-44959392$ & Moderate & Forward & DRG Family regulatory protein \\
\hline & ChrC01g004357 & $48588162-48594007$ & Moderate & Forward & SWEET sugar transporter \\
\hline \multirow{4}{*}{ qLTGC1-1 } & ChrC01g004359 & $48596431-48597483$ & Moderate & Forward & PHD-fnger \\
\hline & ChrC01g004400 & $49078002-49082772$ & Moderate & Forward & Plant invertase/pectin methylesterase inhibitor \\
\hline & ChrC01g004405 & $49166002-49166734$ & Moderate & Reverse & - \\
\hline & ChrC01g004406 & $49193433-49194020$ & Moderate & Forward & - \\
\hline
\end{tabular}

China is one of origin centers of rapeseed; Wild rape in Yunnan Brassica L. is divided from 600 2300 meters above sea level, while mustard-type wild rape is distributed from 1550 2150 meters. The complex ecological environment of Yunnan forms rich and colorful rapeseed germplasm resources, especially the late autumn to early winter sowing contains many cold-resistant germplasm resources during the germination period. Yunnan cabbage rapeseed and mustard rapeseed are mainly distributed in 49 and 43 counties and cities, respectively; Low temperature occurs in late-autumn or early-winter quickly afects rapeseed germination for rapeseed in China, especially the winter sowing rapeseed germination period of 1900-2300 meters in Yunnan Province is usually at low temperature $\left(5-8^{\circ} \mathrm{C}\right) \square$ However, two QTL (qLTGA9-1 and qLTGC1-1) were identifed from 'Huyou21' with cold tolerance of seed germination. At low temperature $\left(8^{\circ} \mathrm{C}\right)$, Huyou21 exhibited excellent tolerance to cold stress, whether the cold tolerance gene at germination of this cultivar is cold domesticated or derived from the wild rape gene is an interesting scientific question. We believe that further research can refer to cold tolerance genes in rice, based on near-isogenic line of cold tolerance at booting stage in rice, such as standing variation of CTB2 and de novo mutation of CTB4a (encoding a conserved leucine-rich repeat receptor-like kinase) underwent stepwise selection to facilitate cold adaptation to expand rice cultivation from high-altitude to high-latitude regions[2]; Upregulation of CTB4a correlates with increased ATP synthase activity, ATP content, enhanced seed setting and improved yield under cold stress conditions[3]. The bZIP73 and OsAPX1 differentiating inter-subspecies evolved directly from wild rice, whereas the novel mutations CTB4a and Ctb1 arose in Geng during adaptation to colder climates[3]. Therefore, improved cold tolerance and expanded cultivation to high-altitude or high-latitude regions would help meet growing rapeseed demand.

\section{References}

1. Zhu J, Wang W, Jiang M, Yang L, Zhou X. QTL mapping for low temperature germination in rapeseed. Sci Rep. 2021,11(1):23382. doi: 10.1038/s41598-021-02912-w. 
2. Zhang Z, Li J, Pan Y, Li J, Zhou L, Shi H, Zeng Y, Guo H, Yang S, Zheng W, Yu J, Sun X, Li G, Ding Y, Ma L, Shen S, Dai L, Zhang H, Yang S, Guo Y, Li Z. Natural variation in CTB4a enhances rice adaptation to cold habitats. Nat Commun. 2017,8:14788. doi: 10.1038/ncomms14788.

3. Li J, Zeng Y, Pan Y, Zhou L, Zhang Z, Guo H, Lou Q, Shui G, Huang H, Tian H, Guo Y, Yuan P, Yang H, Pan G, Wang R, Zhang H, Yang S, Guo Y, Ge S, Li J, Li Z. Stepwise selection of natural variations at CTB2 and CTB4a improves cold adaptation during domestication of japonica rice. New Phytol. 2021,231(3):10561072. doi: 10.1111/nph.17407. 